\title{
Aleukemic extramedullary T lymphoid/myeloid bilineage hematopoietic and lymphoid malignancy with progression to bilineage leukemia at relapse: A case report
}

\author{
MENGYAO WU ${ }^{1}$, XIAOQIU LI ${ }^{2}$, FENG TANG $^{3}$, PING ZHU ${ }^{1}$, TIANLING DING ${ }^{1}$, YAN YUAN $^{1}$ and TONG CHEN ${ }^{1}$ \\ ${ }^{1}$ Department of Hematology, Huashan Hospital, Fudan University, Shanghai 200040; \\ ${ }^{2}$ Department of Pathology, Shanghai Cancer Center, Fudan University, Shanghai 200032; \\ ${ }^{3}$ Department of Pathology, Huashan Hospital, Fudan University, Shanghai 200040, P.R. China
}

Received January 16, 2016; Accepted July 27, 2017

DOI: $10.3892 / \mathrm{ol} .2017 .7212$

\begin{abstract}
Bilineage T lymphoid and myeloid (T/My) neoplasms are rare entities among the hematopoietic and lymphoid malignancies. The majority of patients present with leukemic symptoms in which blasts are observed in the peripheral blood (PB) or bone marrow (BM) at a percentage of $>20 \%$ of nucleated cells. Only a minimal number of cases of T/My bilineage hematopoietic and lymphoid malignancy have been reported with extramedullary infiltration as the initial symptom. The origin of the neoplastic cells in T/My bilineage malignancy has been documented as the hematopoietic stem cells. The present study reports the case of a 31-year-old man with a T/My bilineage malignancy, which initially showed cervical lymph node enlargement beyond the diagnostic criteria of leukemia in the $\mathrm{PB}$ and in the BM. Two distinct malignant populations were detected in the cervical lymph node and pleural effusion, one of which was positive for MPO-staining, while the other was positive for cytoplasmic cluster of differentiation 3. Mutations in platelet-derived growth factor receptor $\alpha$, platelet-derived growth factor receptor $\beta$, fibroblast growth factor receptor 1 and other chromosome abnormalities were excluded. The patient obtained complete remission after conventional chemotherapy, but relapsed with bilineage leukemia within a short period of time. Lymphoid and myeloid lineages have been reported to be differentiated from multipotent progenitors asymmetrically. However, the cellular mutation stage in T/My bilineage
\end{abstract}

Correspondence to: Professor Tong Chen or Dr Yan Yuan, Department of Hematology, Huashan Hospital, Fudan University, 12 Wulumuqi Middle Road, Building No. 2, Shanghai 200040, P.R. China

E-mail: chentong@fudan.edu.cn

E-mail: yuanyan@fudan.edu.cn

Key words: mixed-phenotype acute leukemia, T lymphoid/myeloid, extramedullary infiltration, $\mathrm{T}$ stem cell leukemia/lymphoma, early T-cell precursor-acute lymphoblastic leukemia, hematopoietic differentiation, lineage commitment malignancy remains unclear. The present study also reviews the origin, development and therapeutic strategies for extramedullary T/My bilineage malignancy.

\section{Introduction}

The diagnosis and classification of acute leukemia rely on an array of multidisciplinary approaches, including analyses of morphology, immunophenotype, cytogenetics and molecular genetics. Using these criteria, the majority of acute leukemia cases can be assigned into a specific lineage (1). However, there is a rare type of acute leukemia that exhibits concurrent characteristics of myeloid (My) and T- or B-lymphoid lineages. In the 2008 WHO classification (2), this type of leukemia was termed leukemia of ambiguous lineage, which includes acute undifferentiated leukemia and mixed-phenotype acute leukemia (MPAL) (3).

MPAL is defined as a type of acute leukemia expressing antigens of more than one lineage, which is impossible to assign to any one lineage with certainty (4). The diagnosis of MPAL should exclude cases either by genetic or by clinical features that can be classified into another category. For example, acute myeloid leukemia (AML) with $\mathrm{t}(8 ; 21), \mathrm{t}(15 ; 17)$ and inv(16) can also express lymphoid-associated markers (1). In addition, MPALs with rearrangement of $\mathrm{t}(9 ; 22)(\mathrm{q} 34 ; \mathrm{q} 11.2)$ or $\mathrm{t}(\mathrm{v} ; 11 \mathrm{q} 23)$ should be diagnosed as MPALs with BCR, RhoGEF and GTPase activating protein-ABL proto-oncogene 1 , non-receptor tyrosine kinase (BCR-ABL1) or mixed-lineage leukemia (MLL) rearrangement (1). MAPLs can be classified as bilineage and biphenotypic acute leukemias (4). Bilineage acute leukemias usually contain two distinct blast populations, each of which expresses lymphoid or myeloid lineage markers. In biphenotypic acute leukemia, the blast cells are characterized as one population that expresses myeloid and T- or B-lymphoid markers $(3,5)$. The association and differentiation between bilineage and biphenotypic leukemia remains unclear.

Bilineage T lymphoid and myeloid (T/My) malignancy is rare. To the best of our knowledge, between 1988 and 2015, only 9 publications with a total 18 cases of T/My bilineage MPALs were reported on PubMed (https://www.ncbi.nlm.nih. 
gov/pubmed) (6-14). In the majority of cases, leukemic blast cells were detected initially in the peripheral blood (PB) and bone marrow (BM), meeting the diagnostic criteria of leukemia. Only 2 cases of T/My bilineage malignancy presenting initially with extramedullary infiltration were reported $(15,16)$. The present study reports the case of a 31-year-old man with bilineage T/My malignancy who initially presented with cervical lymph node enlargement beyond the diagnosis of leukemia in the PB and in the BM. The present study also reviews the cellular origin, development and therapeutic strategies of extramedullary T/My bilineage malignancy.

\section{Case report}

A 31-year-old man with painless enlargement of the cervical lymph node was admitted to a local hospital in November 2014. At 2 months prior to admission, the patient had experienced a sore throat with dysphagia. The subjective pain disappeared and the sense of dysphagia was all alleviated following antibiotic treatment. By the end of October 2014, a dry cough without sputum plus stretch pain on the upper breast was noted. The patient was diagnosed with pharyngitis in a local hospital and was treated again with antibiotics. However, no improvement was obtained. The patient was transferred to another hospital and lymph node enlargement was detected in the bilateral cervical and bilateral supraclavicular regions by ultrasonic examination. Visible blood flow in these enlarged lymph nodes was detected by color Doppler flow imaging. The patient then underwent an ultrasound-guided fine-needle aspiration biopsy. A large number of lymphocytes with nuclear granular chromatin and nuclear division were found. Chest computed tomography (CT) revealed multiple enlarged lymph nodes in the regions of the mediastinum, retroperitoneum and each side of the neck. Positron emission tomography (PET)-CT scans detected multiple lymphadenopathy at the bilateral cervical, supraclavicular, thoracic entrance, mediastinum and post-peritoneal areas, with increased fluorodeoxyglucose (FDG) uptake. Few effusions were observed in right pleural cavity. The patient was suspected as having non-Hodgkin's lymphoma and underwent a right cervical lymph node biopsy.

Immunohistochemical (IHC) analysis was then used to examine protein expression. The lymph node sample was fixed in $10 \%$ formalin for $>6 \mathrm{~h}$ at $25^{\circ} \mathrm{C}$, then embedded in paraffin wax for further hematoxylin and eosin (HE) and IHC staining. Thin sections $(4-5-\mu \mathrm{m}$ thick) of paraffin-embedded tissue were adhered to slides. To prevent non-specific binding of the antibody to the tissue, each section was blocked with $1 \%$ rabbit serum (Abcam, Cambridge, UK., Cat. No. ab7487) for $10 \mathrm{~min}$ at $25^{\circ} \mathrm{C}$. Primary antibodies (all primary antibodies are listed below) were added to each section and incubated for $30 \mathrm{~min}$ at $25^{\circ} \mathrm{C}$, then Bond Polymer Refine Detection reagent (Leica Biosystems, Newcastle, UK, cat. no. DS9800) was added for $16 \mathrm{mins}$ at $25^{\circ} \mathrm{C}$. The sections were then incubated with 3,3'-Diaminobenzidine (DAB) system (Maxim Biotech, Inc., Rockville, MD, USA; cat. no. DAB-1031) and then stained with $0.1 \%$ hematoxylin for $5 \mathrm{~min}$ at $25^{\circ} \mathrm{C}$. All slides were observed under a light microscope.

The primary antibodies applied in diagnosis of this patient: Anti-cluster of differentiation (CD)2 (cat. no. MAB-0207, dilution 1:100), anti-CD3 (cat. no. Kit-0003, dilution 1:100), anti-CD4 (cat. no. RMA-0620, dilution 1:100), anti-CD5 (cat. no. Kit-0033, dilution 1:50), anti-CD7 (cat. no. RMA-0739, dilution 1:100), anti-CD8 (cat.no. RMA-0514, dilution 1:100), anti-CD10 (cat. no. MAB-0668, dilution 1:200), anti-CD20 (cat. no. Kit-0001, dilution 1:600), anti-CD34 (cat. no. Kit-0004, dilution 1:400), anti-CD43 (cat. no. MAB-0032, dilution 1:200), anti-CD56 (cat. no. Kit-0028, dilution 1:200), anti-CD68 (cat. no. Kit-0026, dilution 1:400), anti-CD79a (cat. no. MAB-0258, dilution 1:200), anti-CD99 (cat. no. MAB-0059, dilution 1:200), anti-CD117 (cat. no. Kit-0029, dilution 1:200), anti-Bcl-2 (cat. no. MAB-0014, dilution 1:100), anti-chromogranin A (cat. no. MAB-0202, dilution 1:400), anti-epithelial membrane antigen (cat. no. Kit-0011, dilution 1:200), anti-Epstein-Barr virus latent membrane protein 1 (cat. no. MAB-0063, dilution 1:100), anti-Ki-67 (cat. no. MAB-0672, dilution 1:100), anti-myeloperoxidase polyclonal antibody (cat. no. RAB-0379, dilution 1:400), anti-paired box protein (Pax-5) (cat. no. MAB-0706, dilution 1:50), anti-thyroid transcription factor-1 (TTF-1) (cat. no. MAB-0599, dilution 1:200) (all Maxim Biotech, Inc.) and anti-terminal deoxynucleotidyl transferase polyclonal antibody (Ascend Biotechnology, Guangzhou, China, cat. no. AP0221, dilution 1:100),

IHC staining results assessment: the cells without brown color were determined as negative (-), the cells with light brown color were determined as semi-positive (+/-), the cells shown brown color were determined as positive $(+)$.

Immunohistochemical analysis of the cervical lymph node showed myeloperoxidase (MPO)-positive neoplastic cells that were CD3-, CD4-, CD20-, CD79a-, Epstein-Barr virus latent membrane protein $1^{-}$, paired box protein $\mathrm{Pax}-5^{-}$, epithelial membrane antigen ${ }^{-}, \mathrm{Ki}^{-67^{+}}(90 \%)$, terminal deoxynucleotidyl transferase $\left(\mathrm{TdT}^{-}, \mathrm{CD}^{+/-}, \mathrm{CD}^{-}{ }^{-}, \mathrm{CD}^{-} 4^{-}, \mathrm{CD} 117^{-}, \mathrm{CD}^{-}\right.$, chromogranin $\mathrm{A}^{-}$, thyroid transcription factor- $1^{-}$and $\mathrm{B}$-cell lymphoma- $2^{+}$. BM aspirate showed high lymphocytic proliferation with the existence of prolymphocytes.

For flow cytometric analysis of cell membrane antigens, bone marrow and hydrothorax cells were directly incubated with antibodies [anti-CD2-Fluorescein isothiocyanate (FITC; cat. no. 347593), anti-CD3-Phycoerythrin (PE; cat. no. 347347), anti-CD3- Allophycocyanin (APC; cat. no. 340440), anti-CD5-FITC (cat. no. 347303), anti-CD7-FITC (cat. no. 347483), anti-CD7-PE (cat. no. 340581), anti-CD8-FITC (cat. no. 347313), anti-CD10-PE (cat. no. 340921), anti-CD11b-APC (cat. no. 340937), anti-CD13-PE (cat. no. 347837), anti-CD15-FITC (cat. no. 332778), anti-CD16-FITC (cat. no. 335035), anti-CD22-FITC (cat. no. 347573), anti-CD25-FITC (cat. no. 347643), anti-CD33-PE (cat. no. 347787), anti-CD34-APC (cat. no. 340441), anti-CD38-APC (cat. no. 345807), anti-CD45-Peridinin chlorophyll protein (PerCP) (cat. no. 347464), anti-CD56-PE (cat. no. 347747), anti-CD64-PE (cat. no. 644385), anti-CD79a-PE (cat. no. 340579), anti-MPO-FITC (cat. no. 340580), anti-MPO-PE (cat. no. 341642), anti-HLA-DR-PE (cat. no. 347367), anti-TdT-FITC (cat. no. 347194), anti-T cell receptor (TCR)- $\alpha \beta$-FITC (cat. no. 347773), anti-TCR- $\gamma \delta-\mathrm{PE}$ (cat.no.347907) (all BD Biosciences)] for $15 \mathrm{~min}$ at $22^{\circ} \mathrm{C}$ in dark, then FACS lysing solution (BD Biosciences, Franklin Lakes, NJ, USA, cat. no. 349202) was added to lyse red blood cells. 

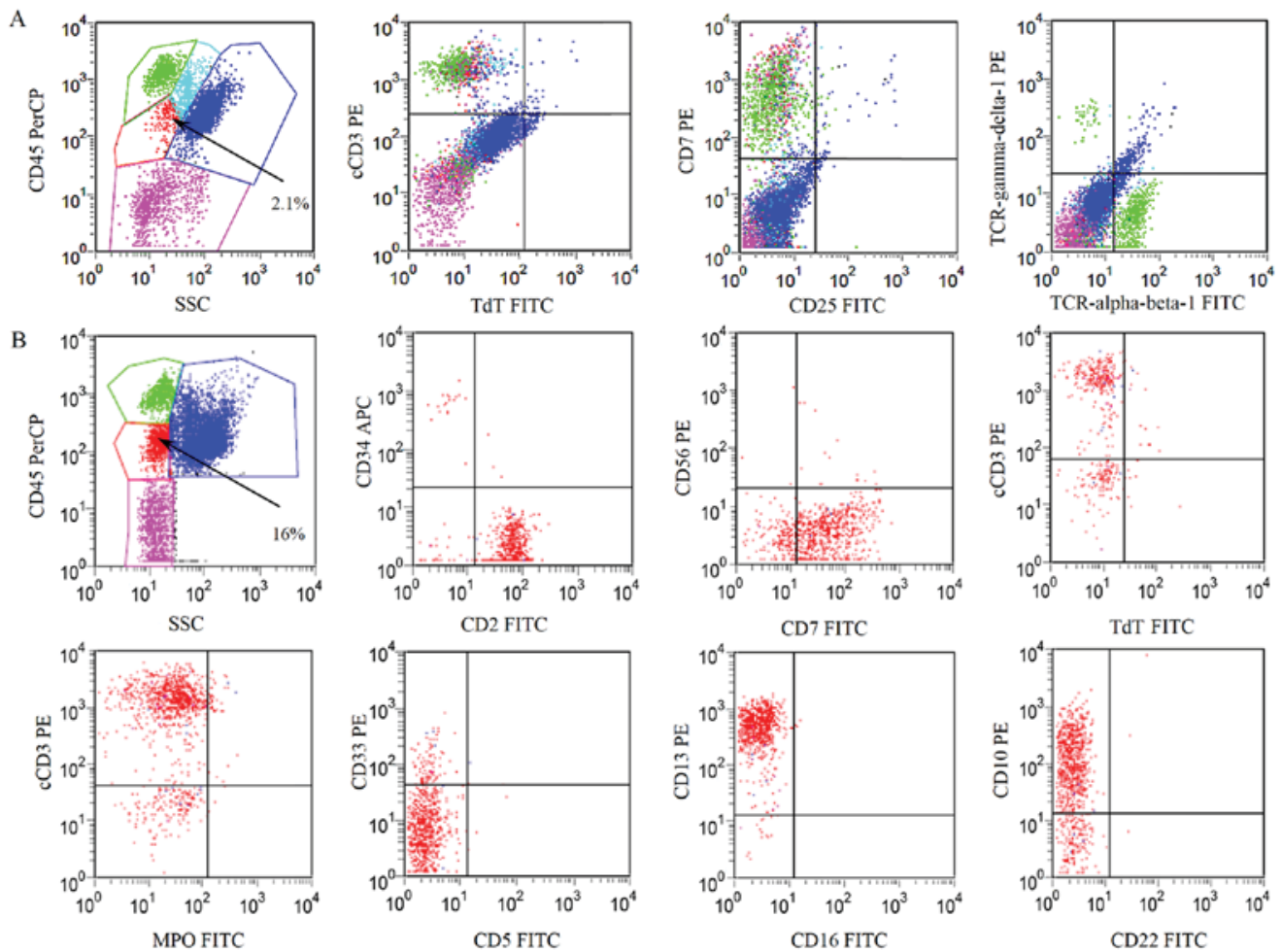

Figure 1. Immunophenotype expression profile of abnormal cells in the BM. Fluorescence-activated cell sorting analysis indicated that phenotypically abnormal nucleated cells (red dots) in the BM were present at (A) 2.1\% in November 2014 and (B) 16\% in December 2014, with expression of CD2, CD7, CD10, CD13 and cyCD3, but without the expression of TCR $\alpha$, TCR $\beta$, TCR $\gamma$ and TCR $\delta$. CD, cluster of differentiation; cyCD3, cytoplasmic CD3; MPO, myeloperoxidase; TdT, terminal deoxynucleotidyl transferase; TCR, T cell receptor; PerCP, peridinin chlorophyll protein complex; SSC, side scatter channel; FITC, fluorescein isothiocyanate; PE, phycoerythrin; APC, allophycocyanin; BM, bone marrow.

Following two washes with PBS, the cells were resuspended and detected by FACSCanto II (BD Biosciences). The corresponding

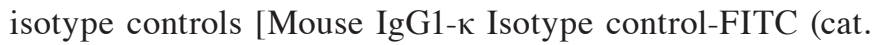
no. 555748), Mouse IgG1-к Isotype control-PE (cat. no. 555749), Mouse IgG1-א Isotype control- PerCP (cat. no. 559425), Mouse

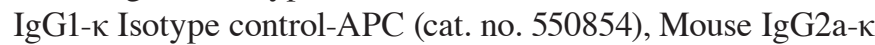
Isotype control-FITC (cat. no. 553456), Mouse IgG2a-k

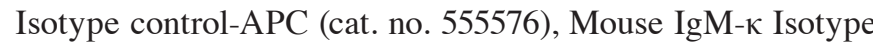
control-FITC (cat. no. 555583) (all BD Biosciences)] were used to avoid non-specific binding of the mouse original antibodies to sample cells in the analysis. FACS data were analyzed by BD FACSDiva software v.8.0.1 (BD Biosciences).

Flow cytometric analysis of intracytoplasmic (cyCD3 and MPO) or intranuclear (terminal deoxynucleotidyl transferase) antigens, bone marrow and hydrothorax cells were fixed and permeabilized with the FIX \& PERM kit (Caltag; Thermo Fisher Scientific, Inc., Waltham, MA, USA; cat. no. GAS-003) according to the manufacturer's protocol, and incubated with primary antibodies for $15 \mathrm{~min}$ at $22^{\circ} \mathrm{C}$ in the dark. After washing twice with PBS, the cells were resuspended and detected using a FACSCanto II flow cytometer (BD Biosciences). FACS data were analyzed by BD FACSDiva software v. 8.0.1 (BD Biosciences).

Flow cytometric analysis of BM cells revealed that $3 \%$ of the nuclear cells were blast cells, $0.9 \%$ of which were myeloblasts with positive staining of CD34 and CD13. The rest of the blast cells in the BM were immature $\mathrm{T}$ lymphocytes expressing $\mathrm{CD} 2, \mathrm{CD} 7, \mathrm{CD} 10, \mathrm{CD} 13, \mathrm{CD} 38$ and cyCD3 (cytoplasmic CD3) (Fig. 1A). The patient was diagnosed with myeloid sarcoma
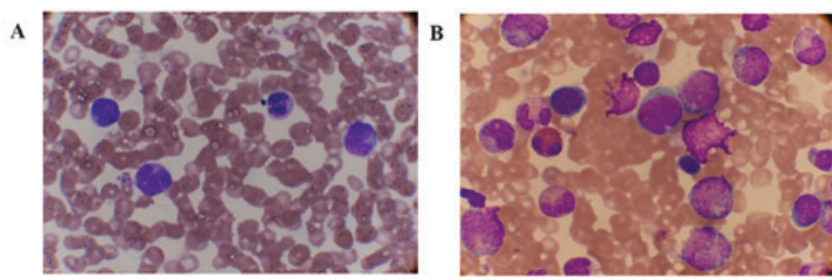

c
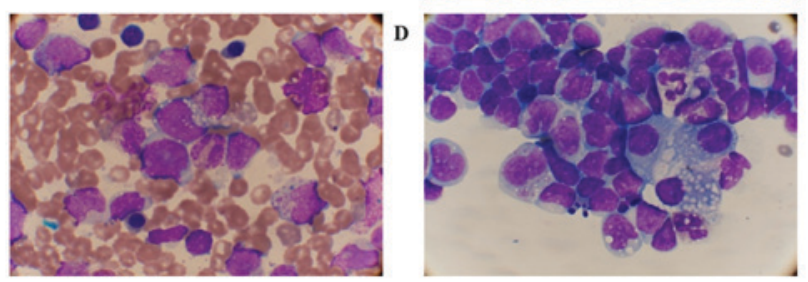

Figure 2. Cellular morphology of the PB, BM and hydrothorax smear (Wright stain). (A) Mature monocytes with abnormal-shaped nuclei and $1 \%$ myelocytes and metamyelocytes were observed in the PB (magnification, $\mathrm{x} 1,000$ ). (B) $2 \%$ myeloblasts, $17 \%$ promyelocytes and (C) $2 \%$ prolymphoid cells (arrow) were observed in the BM (magnification, $\mathrm{x} 1,000$ ). (D) Lymphocytes and monocytes with morphological variation were observed in the smear of the hydrothorax (magnification, x1,000). PB, peripheral blood; BM, bone marrow.

and transferred to Huashan Hospital (Shanghai, China) for further diagnosis and treatment in December 2014.

Upon admission, the physical examination revealed a body temperature of $36.5^{\circ} \mathrm{C}$ and a body weight of $78.5 \mathrm{~kg}$. The patient was found to exhibit superficial lymphadenopathy to a size of $24 \times 15 \mathrm{~mm}$ in the right neck region. The enlarged lymph 
A
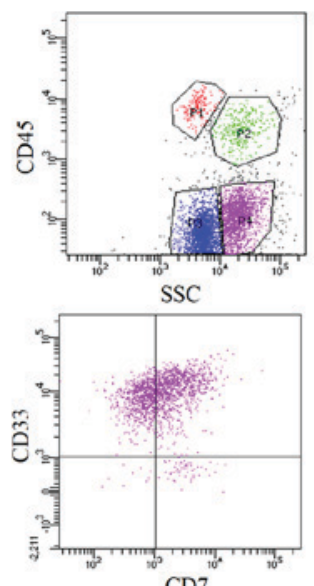

CD7

B

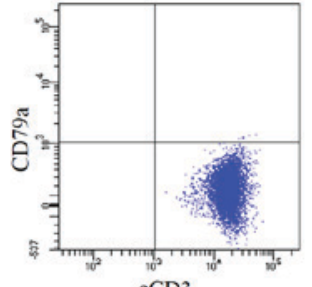

cCD3

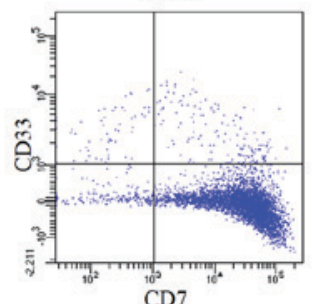

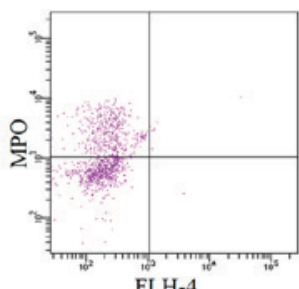

FLH-4

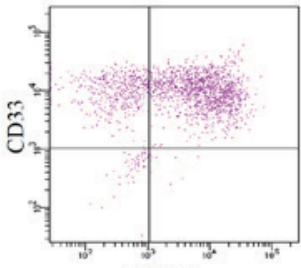

CD11b

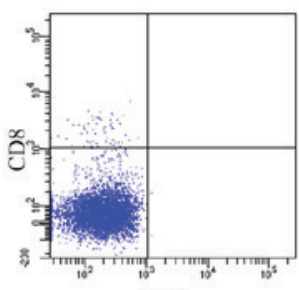

sCD 3

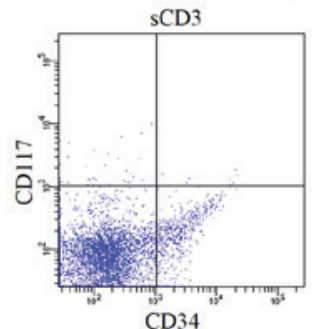

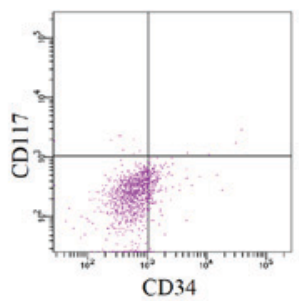

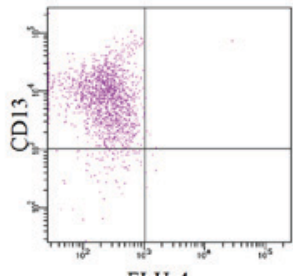

FLH-4

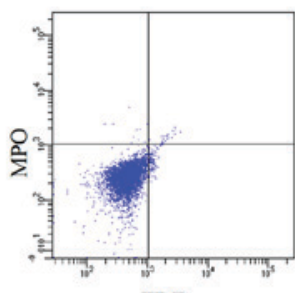

TDT

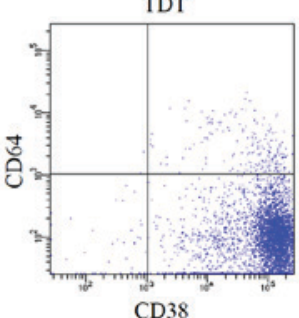

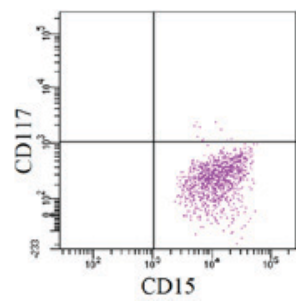

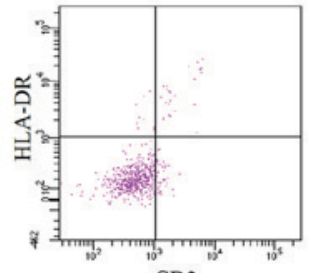

CD2

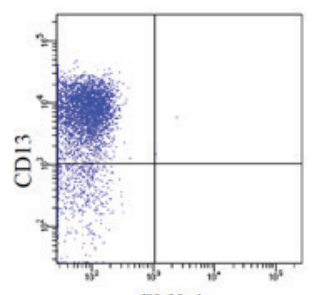

FLH-4

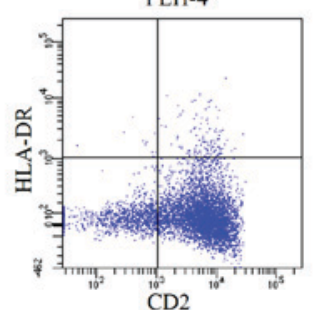

Figure 3. Immunophenotype expression profile of the cells in the thoracic fluid. Fluorescence-activated cell sorting analysis of the cells in the pleural effusion indicated that there were two populations of CD45 low-expression abnormal cells. (A) One expressed myeloid-associated antigens, including MPO, CD15, CD33, CD13 and CD11b ${ }^{\text {dim }}$. (B) The other expressed T lymphoid-associated markers, including cyCD3, CD2 and CD7. CD, cluster of differentiation; MPO, myeloperoxidase; cyCD3, cytoplasmic CD3; HLA-DR, human leukocyte antigen-antigen D-related; SSC, side scatter complex; FLH-4, fluorescence height-4, (no antibody added).

nodes were palpated and appeared tough, with no adhesion to the surrounding tissue, and no broken skin or swelling of the local skin. The patient exhibited no bleeding tendency and no hepatosplenomegaly. Routine blood tests showed $15 \times 10^{9} / 1$

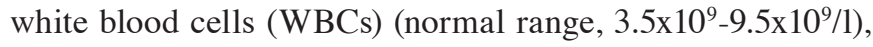
consisting of $35 \%$ neutrophils (normal range, $51-75 \%$ ), $2.0 \%$ eosinophils (normal range, $0.5-5 \%$ ), 38\% monocytes (normal range, 3-8\%) and 18\% lymphocytes (normal range, 20-40\%). The hemoglobin level was $148 \mathrm{~g} / \mathrm{l}$ (normal range, $130-175 \mathrm{~g} / \mathrm{l}$ ) and the platelet count was $145 \times 10^{9} / 1$ (normal range, $\left.125 \times 10^{9}-350 \times 10^{9} / 1\right)$. The serum lactate dehydrogenase (LDH) level was slightly increased at 284 IU/1 (normal range, 125-225 IU/1). A peripheral blood smear detected 39\% mature monocytes with abnormally shaped nuclei. No blast cells, but metamyelocytes, promonocyte-like cells and prolymphoid cells were observed in the PB smear at $1 \%$ of nucleated cells (Fig. 2A). A BM smear found markedly increased nucleated cells at the percentage of $2 \%$ myeloblasts (normal range, $0-1 \%$ ) and $2 \%$ prolymphoids (normal range, $0-1.5 \%$ ) cells (Fig. 2B and $\mathrm{C}$ ). Immunological analysis of the $\mathrm{BM}$ revealed that the percentage of abnormal cells increased to $16 \%$ of nucleated cells, with expression of CD2, CD7, CD10, CD13, CD38 and cyCD3 (Fig. 1B). Moderate right pleural effusion was detected in the patient. The cellular hydrothorax smear demonstrated a large number of lymphocytes and monocytes with morphological variation (Fig. 2D). The cellular immunophenotypic analysis of the pleural effusion indicated that there were two populations of CD45 low-expression abnormal cells. One expressed myeloid-associated antigens, including MPO, CD15, CD33, CD13 and CD11b ${ }^{\text {dim }}$ (Fig. 3A). The other expressed T lymphoid-associated markers, including cyCD3, CD2 and CD7 (Fig. 3B). The two populations were each found to have minimal co-expression of markers specific to their counterpart. $\mathrm{CyCD}^{+}$cells co-expressed myeloid marker CD13, while $\mathrm{MPO}^{+}$cells co-expressed lymphoid marker CD7. The two populations were CD34- and CD117- (Fig. 3).

The pathological cervical lymph node sample was reexamined. Coexistence of $\mathrm{CD}^{+} / \mathrm{CD}^{+} / \mathrm{CD} 2^{+} \mathrm{T}$ lymphoid and $\mathrm{MPO}^{+}$myeloid neoplastic cells was revealed under the microscope in the same region (Fig. 4A-D). Myeloid neoplastic cells showed pleomorphic nuclei and distinct nucleoli with positive immunostaining for MPO. Lymphoid neoplastic cells showed ovoid nuclei, finely dispersed chromatin and inconspicuous nucleoli, with positive immunostaining of lymphoid-associated marker, containing CD3, CD4, CD99, CD43 and TdT (Fig. 4E-M). The boundary between two 

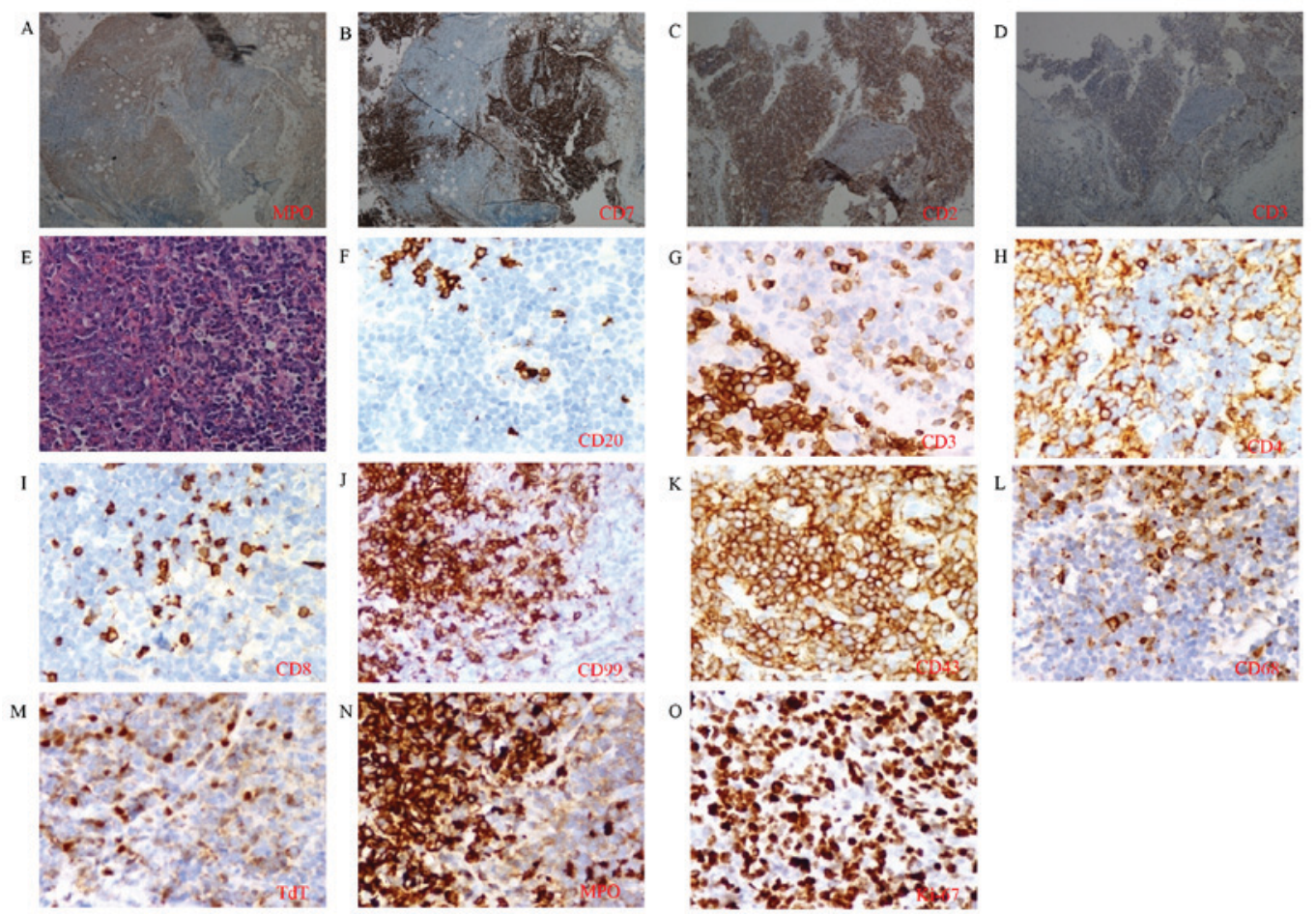

Figure 4. Immunohistological analysis of the cervical lymph node. Coexistence of $\mathrm{T}$ lymphoid and myeloid neoplastic cells was found in the same region by staining for (A) MPO, (B) CD7, (C) CD2 and (D) CD3 (magnification, x100). (E) Cellular morphology was distinguishable by hematoxylin and eosin staining (magnification, x400). Expression of (F) CD20, (G) CD3, (H) CD4, (I) CD8, (J) CD99, (K) CD43, (L) CD68, (M) TdT, (N) MPO and (O) Ki-67 at high magnification was detected (magnification, $\mathrm{x} 400$ ). $\mathrm{CD}$, cluster of differentiation; MPO, myeloperoxidase; TdT, terminal deoxynucleotidyl transferase.

populations was distinguishable by staining for MPO (Fig. 4A and $\mathrm{N}$ ) and CD3 (Fig. 4D and G). The two populations exhibited high expression of Ki-67 (Fig. 4O). Cytogenetic analysis found that $1 / 20$ of the examined BM cells possessed the translocation $\mathrm{t}(12 ; 13)(\mathrm{q} 10 ; \mathrm{p} 10)$, while the remainder were of a normal karyotype. Investigation into genetic mutations associated with acute leukemia, including AML12/ETO, PML/RAR $\alpha$, PLZF/RAR $\alpha$, NPM/RAR $\alpha$, CBF $\beta / M Y H 11$, TLS/ERG, DEK/CAN, NPM/MLF1, dupMLL, MLL/AF6, MLL/AF9, MLL/AF10, MLL/AF17, MLL/ELL,EVI1,HOX11, FIP1L1/PDGFR $\alpha$, ETV6/PDGFR $\beta$ and BCR/ABL (forms P190, P210 and P230), was negative in the BM. Detection of T-cell receptor (TCR) rearrangement by polymerase chain reaction analysis and FGFR1 mutation by fluorescence in situ hybridization, as described previously $(17,18)$, detection in lymph node samples were negative.

For PCR, total RNA of the bone marrow sample was extracted using RNAprep Pure Blood kit (Tiangen Biotech, Beijing, China, cat. no. DP433), and reverse-transcribed to cDNA using the Reverse Transcription System kit (Promega Corporation, Madison, WI, USA), according to the manufacturer's protocol. For $20 \mu \mathrm{l}$ PCR system, $10 \mu \mathrm{l}$ GoTaq GreenMaster mix (Promega Corporation), $1.5 \mu 1$ cDNA, $1.5 \mu 1$

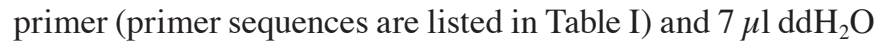
was added. Following PCR amplification (thermocycling conditions are listed in Table I) of the target gene, gene expression was analyzed by DNA gel electrophoresis. Total DNA of paraffin-embedded lymph node tissue sample was extracted using a QIAamp DNA Mini kit (Qiagen GmbH, Hilden, Germany). For $20 \mu 1$ PCR system, $10 \mu 1$ GoTaq GreenMaster mix (Promega Corporation), $2 \mu \mathrm{l}$ DNA, $2 \mu \mathrm{l}$ primer mix and
$6 \mu \mathrm{ld} \mathrm{dH}_{2} \mathrm{O}$ were added. Following PCR amplification of the target gene, the gene expression was analyzed by DNA gel electrophoresis.

The patient was diagnosed with a hemato-lymphoid neoplasm showing separate differentiation toward $\mathrm{T}$ lymphoblastic and myeloid lineage, and was administered a combined induction chemotherapy regimen (chemotherapy cycle 1 : $4 \mathrm{mg} /$ day vindesine intravenous on day $1,10 \mathrm{mg} /$ day idarubicin intravenous on days $1-3,180 \mathrm{mg} /$ day cytarabine intravenous on days 1-7 and $80 \mathrm{mg} /$ day methylprednisolone intravenous on days 1-7) in December 2014. Subsequently, 1 day after the final dose of chemotherapy, the chest CT scan indicated that the pleural effusion was significantly reduced. The superficial lymph nodes shrank and the cough symptom improved. The number of WBCs and the proportion of monocytes decreased to $6.99 \times 10^{9} / 1$ and $4 \%$, respectively. The patient then received chemotherapy of hyper-CVAD A regimen (chemotherapy cycle 2: $1 \mathrm{~g} /$ day cyclophosphamide intravenous on days 1-3, $4 \mathrm{mg} / \mathrm{day}$ vindesine intravenous on days 4 and $11,15 \mathrm{mg} / \mathrm{day}$ idarubicin intravenous on days $4,40 \mathrm{mg} / \mathrm{day}$ dexamethasone intravenous on days 1-4 and 11-14), hyper-CVAD B regimen (chemotherapy cycle 3: $1.8 \mathrm{~g} /$ day methotrexate intravenous on day 1, $6 \mathrm{~g}$ /day cytarabine intravenous on days 2-3) and the induction regimen (chemotherapy cycle 4: identical to cycle 1) repeatedly as consolidation treatment. Immediately prior to the 4th cycle of chemotherapy, PET-CT was reviewed and all the enlarged lymph nodes had normalized. A hypermetabolic lesion in the mediastinum remained detectable, but at a markedly decreased level compared with that previously. The proportion of abnormal $\mathrm{T}$ cells in the BM was reduced to $0.3 \%$. The matched unrelated allogeneic hematopoietic stem 


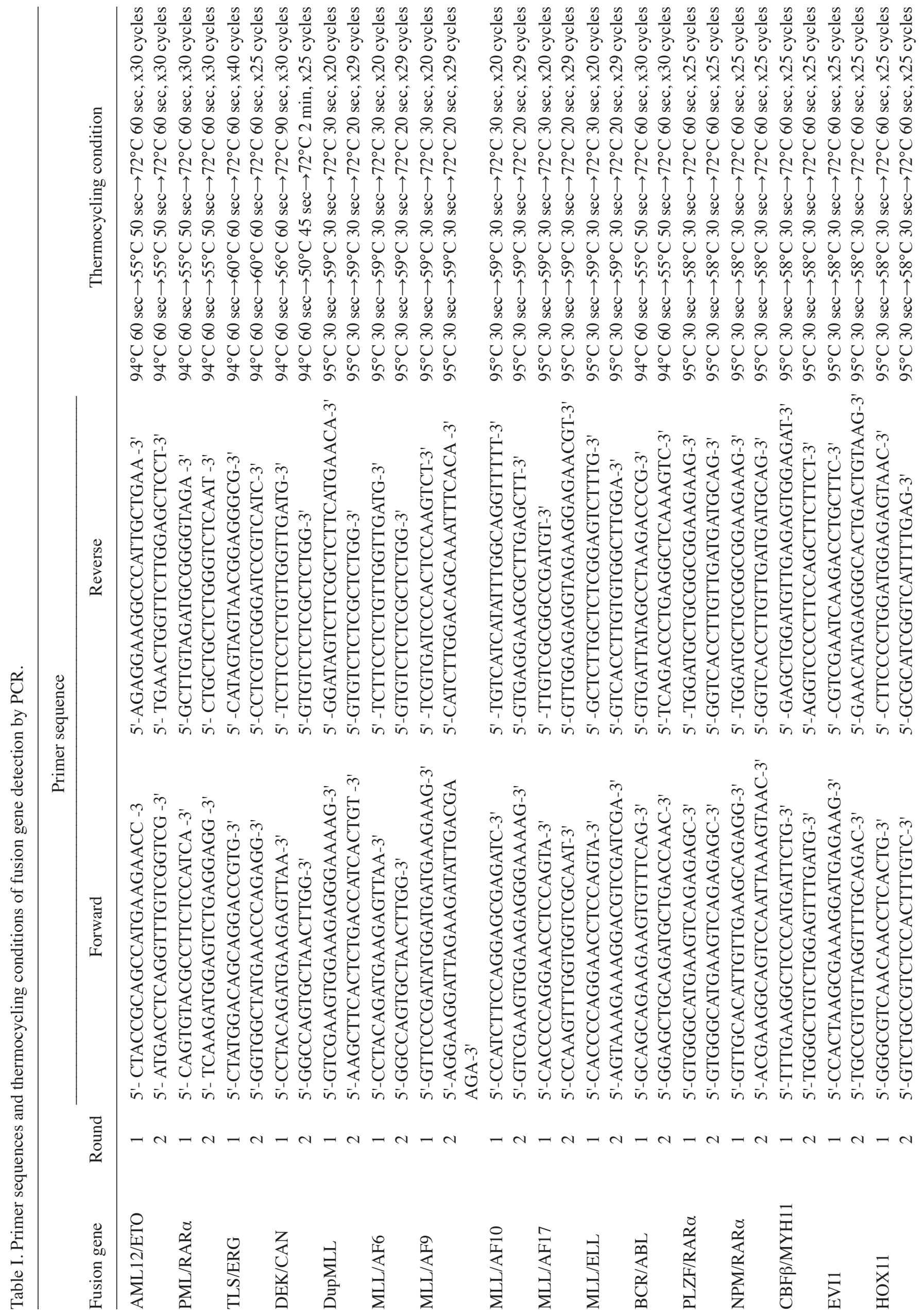


cell transplantation (allo-HSCT) was postponed on account of invasive Cryptococcus infection in the right lung. However, in late June 2015, the neoplasm relapsed with leukemic symptoms; $24 \%$ of abnormal $\mathrm{T}$ cells and $44 \%$ of CD45 myeloid blasts were detected in the $\mathrm{BM}$, with infiltrations in the cervical lymph nodes and the posterior pharyngeal wall. The repeat use of the initial induction regimen and more intensive regimen $(4 \mathrm{mg} / \mathrm{day}$ vindesine intravenous on days 1 and 8 , $12 \mathrm{mg} /$ day mitoxantrone intravenous on days $1-3,1 \mathrm{~g}$ /day cytarabine intravenous on days 1, 3 and 5, and methylprednisolone intravenous $60 \mathrm{mg}$ /day on days $1-9,40 \mathrm{mg} /$ day on days $10-15$ and 3,750 $\mathrm{U}$ peg-asparaginase intramuscular on day 15 ), all failed. The patient succumbed in mid-September 2015.

The requirement for patient consent for publication was waived by the Institutional Review Board of Huashan Hospital.

\section{Discussion}

The patient in the current study presented with extra-medullary infiltration as the initial symptom. Notably, two distinct neoplastic populations, cyCD3 ${ }^{+} \mathrm{T}$ lymphoid cells and $\mathrm{MPO}^{+}$ myeloid cells, were detected in the pleural effusion and lymph nodes. None of the cells expressed the pluripotent markers CD34 or CD117. A certain minimal degree of cross-expression of markers specific to their lineage counterpart was detected. $\mathrm{CyCD}^{+} \mathrm{T}$ lymphoid cells coexpressed the myeloid antigen $\mathrm{CD13}$, while $\mathrm{MPO}^{+}$myeloid lineage cells coexpressed lymphoid marker CD7. However, the fact that none of the populations at the initial stage fitted the diagnostic criteria of acute leukemia within either the BM or the PB raised a difficulty in providing an accurate diagnosis $(1,4)$. To clarify the origin of the neoplastic cells and the differentiation stage of mutational occurrence should assist in forming an accurate diagnosis and choosing the therapeutic strategy.

The symmetric model of lineage commitment differentiation from HSCs, in which clear division of lymphoid and myeloid commitment is the first step of lineage restriction, used to be widely accepted. In this model, common myeloid progenitors (CMPs) and common lymphoid progenitors (CLPs) are symmetrically derived from the same multipotent progenitors (MPPs). CMPs then gradually differentiate into all types of myeloid offspring, including granulocytes, monocytes, erythrocytes and megakaryocytes. Similarly, CLPs differentiate into mature lymphoid cells, including T cells, B cells and natural killer cells (19).

However, previous studies indicated that HSCs differentiate into lymphoid lineage and myeloid lineages asymmetrically. All MPPs subsets contribute to lymphoid lineage differentiation. The most primitive fms-like tyrosine kinase $3^{\text {lo }} /$ vascular cell adhesion protein $1^{+}\left(\right.$Flt $\left.3^{\text {lo }} \mathrm{VCAM}-1^{+}\right)$ MPPs were indicated to be able to give rise to CLPs and CMPs. However, the most advanced Flt $3^{\text {hi }}$ VCAM-1 $1^{-}$MPPs only gave rise to CLPs $(20,21)$, suggesting that lymphoid differentiation occupies the backbone of the differentiation process, whereas myeloid differentiation is a lateral branch. Two other stages specified as granulocyte/macrophage-lymphoid bipotent progenitors and lymphoid-specified progenitors sit between MPPs and CLPs (22). This model provides certain clues to explain the occurrence of $\mathrm{T} / \mathrm{My}$ bilineage/biphenotype malignancies. 


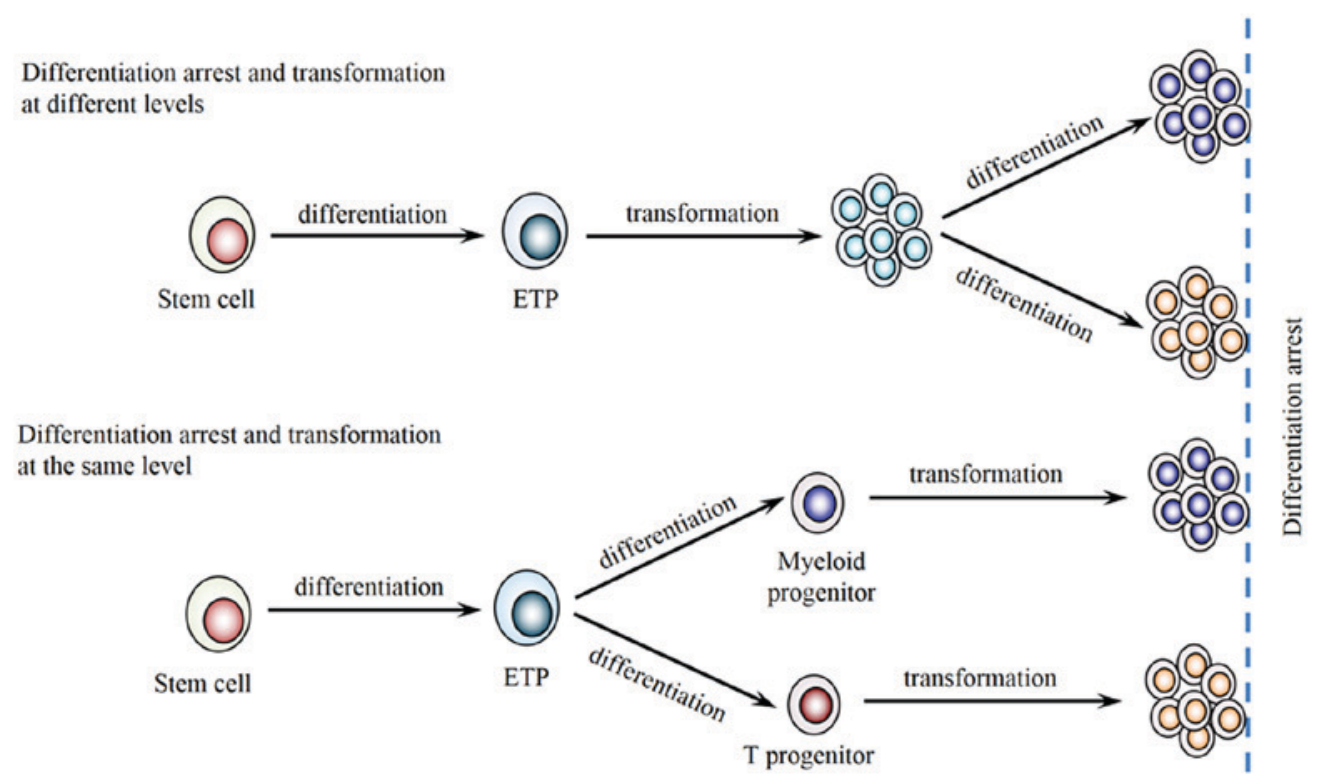

Figure 5. Hypothetical schema of cell transformation and differentiation arrest in bilineal T/My malignancy. The differentiation arrest and transformation can occur at the same level or at different levels. In the current case, we speculated that the neoplastic cells were transformed from early DN2a stage, which retained $\mathrm{T}$ and myeloid differentiation potential, resulting in two distinct populations in the same extramedullary site. T/My, T lymphoid and myeloid; ETP, early T-cell precursor.

Thymic T lymphocytes mature from CD4-CD8- doublenegative (DN) cells to $\mathrm{CD} 4^{+} \mathrm{CD} 8^{+}$double-positive (DP) cells, then to the fully mature CD4 or CD8 single positive (SP) cells. The DN stage can be subdivided into 4 substages depending on the lack of CD4 and CD8 surface expression and differential expression of CD25 and CD44: CD44 ${ }^{+} \mathrm{CD} 25^{-}$(DN1), $\mathrm{CD}_{4} 4^{+} \mathrm{CD} 25^{+}$(DN2), CD44-CD25 ${ }^{+}$(DN3), and CD44-CD25 (DN4). In addition, the differential expression of CD117 in DN2 cells enabled the establishment of two further subsets, DN2a (CD4-CD8 $\left.{ }^{-C D} 44^{+} \mathrm{CD} 25^{+} \mathrm{CD} 117 \mathrm{hi}\right)$ and DN2b (CD4-C D8 ${ }^{-C D} 44^{+} \mathrm{CD} 25^{+} \mathrm{CD} 117$ int $)(23,24)$. In the thymic $\mathrm{T}$ cell maturation process, the myeloid differentiation potential is retained until the middle of the DN2 stage, while the rearrangement of TCR starts from the DN2b stage (25). Meanwhile, the cellular phenotypes were orchestrated with different maturation stages.

In the 1980s and the 1990s, a class of T-ALL with myeloid differentiation features, known as $\mathrm{T}$ stem cell leukemia/lymphoma (T-SCL/lymphoma) was reported in the literature (26-30). T-SCL/lymphoma cells are $\mathrm{CD}^{+} / \mathrm{CD} 4 / \mathrm{CD} 8$, representing a portion of $\mathrm{T}$ lymphocytes that are going to migrate from the BM to the thymus (29). This indicated that the neoplastic cells of T-SCL/lymphoma originated from the HSCs, which maintained the ability to differentiate into lymphoid and myeloid lineages $(26,30)$. In almost all the reported T-SCL/lymphoma cases, the patients presented with a giant mass in the mediastinum or lymph nodes, with coexpression of $\mathrm{T}$ lymphoid and myeloid phenotypes on blast cells $(27,29)$. However, with molecular and immunological progress in the studies of hematopoietic diseases, the diagnostic criteria depending only on the expression of CD7, CD4 and CD8 were far from accurate.

In the 2008 WHO classification (2), T-precursor neoplasms were classified into pro-T, pre-T, cortical $\mathrm{T}$ and medullary $\mathrm{T}$ subtypes according to the neoplastic phenotypes. Expression of cyCD3, CD34, TdT, surface CD3 (sCD3), CD4 and CD8 can assist in distinguishing between $\mathrm{T}$ subtype differentiation stages (31). However, the origin of T/My MPALs remains unclarified.

Since 2009, a novel subtype of T-ALL, early T-cell precursor-acute lymphoblastic leukemia (ETP-ALL), has been reported. ETP-ALL is a high-risk subset and comprises $\sim 15 \%$ of all T-ALL cases (32). ETPs represent thymocytes recently immigrating from the BM to the thymus and thus retaining the multilineage differentiation potential (33-35). ETPs, immunophenotypically characterized as $\mathrm{CD}^{- \text {/weak }}, \mathrm{CD1} \mathrm{a}^{-}$and $\mathrm{CD} 8$, with positive expression of myeloid markers (human leukocyte antigen-antigen D-related, CD13, CD33, CD11b or CD65) and stem cell markers (CD34 and CD117) (36), were indicated to be at the DN1 differentiation stage (25).

With the latest understanding of the T lymphoid differentiation process, we hypothesized that the neoplastic cells in the present case were transformed from early DN2a stage, which retained further differentiation potential into $\mathrm{T}$ lymphoid and myeloid lineages, resulting in two distinct populations in the same extramedullary site (Fig. 5). The patient was thus diagnosed with a hemato-lymphoid neoplasm showing separate differentiation toward $\mathrm{T}$ lymphoblastic and myeloid lineages. The disease progressed to develop leukemic symptoms at relapse, where blasts were detected in the BM and extramedullary site. However, there remains a lack of accurate nomenclature to name this type of T/My bilineage malignancy with extramedullary infiltration at the initial stage. The pathological development mechanism of this hematological malignant entity requires further investigation.

In treating bilineal or biphenotypic MPALs, there are no generally accepted regimens of induction chemotherapy that can cover the lymphoid and/or the myeloid lineage. In a previous study, 20 cases of MPAL, including 1 case of T/My bilineage leukemia, were reported to be treated with prednisone, vincristine, L-asparaginase and daunorubicin, and successfully 
achieved complete remission after initial induction therapy (7). The fludarabine, cytarabine and idarubicine protocol (9) and ALL-based induction chemotherapy (10) were previously reported as used in treating MPALs in different medical centers. However, it has been indicated that a large proportion of MPALs are resistant and refractory to conventional chemotherapy. Allo-HSCT, particularly early in the disease course, is thus far the best treatment strategy (37). Allo-HSCT was delayed in the current patient due to invasive lung Cryptococcus infection. The disease relapsed and progressed rapidly, and the patient did not obtain the opportunity to achieve repeat remission.

In conclusion, the present case initially showed extramedullary infiltration of distinct $\mathrm{T}$ lymphoid and myeloid populations beyond the diagnosis of leukemia in the PB or in the BM. The disease progressed to leukemia at relapse stage, indicating the very early stage of T/My bilineage MPALs. Clarification of the origin of the neoplastic cells and the differentiation stage of mutational occurrence is crucial to choose the appropriate treatment strategy.

\section{Acknowledgements}

The authors would like to thank Kindstar Global Corporation (Shanghai, China) for performing PCR and FISH experiments. The present study was partially supported by the National Natural Science Foundation of China (grant nos 31371480 and 91542109) and the Foundation of the Science and Technology Commission of Shanghai Municipality (grant no. 16XD1400600).

\section{References}

1. Weinberg OK and Arber DA: Mixed-phenotype acute leukemia: Historical overview and a new definition. Leukemia 24: 1844-1851, 2010

2. Swerdlow SH, Campo E, Harris NL, Jaffe ES, Pileri SA, Stein H, Thiele J and Vardiman JW: WHO Classification of Tumours of Haematopoietic and Lymphoid Tissues. 4th edition. IARC, Lyon, pp150-155, 2008.

3. Béné MC and Porwit A: Acute leukemias of ambiguous lineage. Semin Diagn Pathol 29: 12-18, 2012.

4. Béné MC: Biphenotypic, bilineal, ambiguous or mixed lineage: Strange leukemias!. Haematologica 94: 891-893, 2009.

5. Nishiuchi T, Ohnishi H, Kamada R, Kikuchi F, Shintani T, Waki F, Kitanaka A, Kubota Y, Tanaka T and Ishida T: Acute leukemia of ambiguous lineage, biphenotype, without CD34, TdT or TCR-rearrangement. Intern Med 48: 1437-1441, 2009.

6. Tota G, Coccaro N, Zagaria A, Anelli L, Casieri P, Cellamare A, Minervini A, Minervini CF, Brunetti C, Impera L, et al: ADAMTS2 gene dysregulation in T/myeloid mixed phenotype acute leukemia. BMC Cancer 14: 963, 2014.

7. Bachir F, Zerrouk J, Howard SC, Graoui O, Lahjouji A, Hessissen L, Bennani S, Quessar A and El Aouad R: Outcomes in patients with mixed phenotype acute leukemia in Morocco. J Pediatr Hematol Oncol 36: e392-e397, 2014.

8. Sharma P, Lall M, Jain P, Saraf A, Sachdeva A and Bhargava M: A Bi-Lineal acute leukemia (T/Myeloid, NOS) with complex cytogenetic abnormalities. Indian J Hematol Blood Transfus 29: 119-122, 2013.

9. Colovic M, Colovic N, Jankovic G, Kraguljac Kurtovic N, Vidovic A, Djordjevic V and Bogdanovic A: Mixed phenotype acute leukemia of $\mathrm{T} /$ myeloid type with a prominent cellular heterogeneity and unique karyotypic aberration 45, XY, dic(11;17). Int J Lab Hematol 34: 290-294, 2012.

10. Oliveira JL, Kumar R, Khan SP, Law ME, Erickson-Johnson M, Oliveira AM, Ketterling RP and Dogan A: Successful treatment of a child with T/myeloid acute bilineal leukemia associated with TLX3/BCL11B fusion and 9q deletion. Pediatr Blood Cancer 56: 467-469, 2011
11. Weir EG, Ali Ansari-Lari M, Batista DA, Griffin CA, Fuller S, Smith BD and Borowitz MJ: Acute bilineal leukemia: A rare disease with poor outcome. Leukemia 21: 2264-2270, 2007.

12. Kobayashi N, Matsuda K, Sakashita K, Matsuzaki S, Iwasaki R and Koike K: Bilineage acute leukemia of T-lymphoid and myeloid lineages. Haematologica 89: 1139-1141, 2004.

13. Akashi K, Shibuya T, Harada M, Morioka E, Oshima K, Kimura N, Takeshita M, Kurokawa M, Kikuchi M and Niho Y: Acute 'bilineal-biphenotypic' leukaemia. Br J Haematol 74: 402-407, 1990.

14. Akashi K, Harada M, Shibuya T, Morioka E, Okamura T, Asano Y, Taniguchi S, Teshima T, Kikuchi M and Niho Y: Clinical characteristics of hybrid leukemia: Report of five cases. Leuk Res 14: 145-153, 1990.

15. Licci S, Canal F, Dei Tos AP, Fedrigo M, Gherlinzoni F, Brenna A, Zanatta L and Rossi S: Aleukemic granulocytic sarcoma with associated T-cell lymphoblastic lymphoma in the same lymph node: Morphologic features and molecular signatures. Leuk Lymphoma 49: 1411-1415, 2008.

16. Schwarz J, Trnková Z, Bedrlíková R, Jirásek A, Záková D, Trnený M, Sedlácková E, Kodetová D, Valenta J, Stöckbauer $\mathrm{P}$, et al: Aleukemic granulocytic sarcoma with AML1/ETO fusion gene expression and clonal T cell populations. Leuk Res 25: 1137-1142, 2001.

17. van Dongen JJ, Langerak AW, Brüggemann M, Evans PA, Hummel M, Lavender FL, Delabesse E, Davi F, Schuuring E, García-Sanz R, et al: Design and standardization of PCR primers and protocols for detection of clonal immunoglobulin and T-cell receptor gene recombinations in suspect lymphoproliferations: Report of the BIOMED-2 Concerted Action BMH4-CT98-3936. Leukemia 17: 2257-2317, 2003.

18. Patnaik MM, Gangat N, Knudson RA, Keefe JG, Hanson CA, Pardanani A, Ketterling RP and Tefferi A: Chromosome 8p11.2 translocations: Prevalence, FISH analysis for FGFR1 and MYST3, and clinicopathologic correlates in a consecutive cohort of 13 cases from a single institution. Am J Hematol 85: 238-242, 2010.

19. Lai AY and Kondo M: T and B lymphocyte differentiation from hematopoietic stem cell. Semin Immunol 20: 207-212, 2008.

20. Lai AY, Lin SM and Kondo M: Heterogeneity of Flt3-expressing multipotent progenitors in mouse bone marrow. J Immunol 175: 5016-5023, 2005.

21. Lai AY and Kondo M: Asymmetrical lymphoid and myeloid lineage commitment in multipotent hematopoietic progenitors. J Exp Med 203: 1867-1873, 2006.

22. Adolfsson J, Månsson R, Buza-Vidas N, Hultquist A, Liuba K, Jensen CT, Bryder D, Yang L, Borge OJ, Thoren LA, et al: Identification of Flt3+ lympho-myeloid stem cells lacking erythro-megakaryocytic potential a revised road map for adult blood lineage commitment. Cell 121: 295-306, 2005.

23. Koch U and Radtke F: Mechanisms of T cell development and transformation. Annu Rev Cell Dev Biol 27: 539-562, 2011.

24. Ceredig R and Rolink T: A positive look at double-negative thymocytes. Nat Rev Immunol 2: 888-897, 2002.

25. Kawamoto H, Ikawa T, Masuda K, Wada H and Katsura Y: A map for lineage restriction of progenitors during hematopoiesis: The essence of the myeloid-based model. Immunol Rev 238: 23-36, 2010.

26. Nagano M, Kimura N, Akiyoshi T, Nishimura J, Kozuru M, Okamura J, Katsuno M, Yoshida T, Takeshita M, Tachibana $\mathrm{K}$, et al: T-stem cell leukemia/lymphoma with both myeloid lineage conversion and T-specific delta recombination. Leuk Res 21: 763-773, 1997.

27. Katsuno M, Abe Y, Taguchi F, Yufu Y, Sadamura S, Goto T, Takatsuki H, Nishimura J, Hirata J, Akiyoshi T, et al: CD7+ stem cell leukemia/lymphoma. Features of a subgroup without circulating blast cells. Cancer 72: 99-104, 1993.

28. Silva ML, de Oliveira MS, Valente AN, Abdelhay E, Bouzas LF, Laun L and Ribeiro RC: CD7+, CD4-/CD8- acute leukemia with $\mathrm{t}(11 ; 14)(\mathrm{p} 15 ; \mathrm{q} 11)$ in a child. Cancer Genet Cytogenet 56: 171-176, 1991.

29. Kurtzberg J, Waldmann TA, Davey MP, Bigner SH, Moore JO, Hershfield MS and Haynes BF: CD7+, CD4-, CD8- acute leukemia: A syndrome of malignant pluripotent lymphohematopoietic cells. Blood 73: 381-390, 1989.

30. Hershfield MS, Kurtzberg J, Harden E, Moore JO, Whang-Peng J and Haynes BF: Conversion of a stem cell leukemia from a T-lymphoid to a myeloid phenotype induced by the adenosine deaminase inhibitor 2'-deoxycoformycin. Proc Natl Acad Sci USA 81: 253-257, 1984 
31. Onciu M: Acute lymphoblastic leukemia. Hematol Oncol Clin North Am 23: 655-674, 2009.

32. Zhang J, Ding L, Holmfeldt L, Wu G, Heatley SL, Payne-Turner D, Easton J, Chen X, Wang J, Rusch M, et al: The genetic basis of early T-cell precursor acute lymphoblastic leukaemia. Nature 481: 157-163, 2012.

33. Bell JJ and Bhandoola A: The earliest thymic progenitors for T cells possess myeloid lineage potential. Nature 452: 764-767, 2008.

34. Wada H, Masuda K, Satoh R, Kakugawa K, Ikawa T, Katsura Y and Kawamoto $\mathrm{H}$ : Adult T-cell progenitors retain myeloid potential. Nature 452: 768-772, 2008.
35. Rothenberg EV, Moore JE and Yui MA: Launching the T-cell-lineage developmental programme. Nat Rev Immunol 8: 9-21, 2008

36. Coustan-Smith E, Mullighan CG, Onciu M, Behm FG, Raimondi SC, Pei D, Cheng C, Su X, Rubnitz JE, Basso G, et al: Early T-cell precursor leukaemia: A subtype of very high-risk acute lymphoblastic leukaemia. Lancet Oncol 10: 147-156, 2009.

37. Shimizu H, Saitoh T, Machida S, Kako S, Doki N, Mori T, Sakura T, Kanda Y, Kanamori H, Miyawaki S, et al: Allogeneic hematopoietic stem cell transplantation for adult patients with mixed phenotype acute leukemia: Results of a matched-pair analysis. Eur J Haematol 95: 455-460, 2015. 